\section{Evaluación de competencias de intubación traqueal mediante un escenario simulado en internos de medicina}

\author{
MAX ANDRESEN ${ }^{1}$, ARNOLDO RIQUELME ${ }^{2,3}$, PABLO HASBÚN $^{1}$, \\ CARLA DÍAZ $^{5, a}$, RODRIGO MONTAÑA ${ }^{4}$, TOMÁS REGUEIRA ${ }^{1}$
}

\section{Evaluation of competencies for tracheal intubation among medical students}

Background: Chilean medical schools curricula are focused on the acquisition of competencies. Tracheal intubation is considered a terminal competence. Aim: To evaluate the competencies related to airway management including tracheal intubation in undergraduate medical students. Material and Methods: Prospective observational study. Thirty medical students in the sixth year of a seven years undergraduate program (25.2\% of the cohort) were randomly chosen and invited to participate in a simulated environment of elective tracheal intubation using a manikin. The students were assessed according to a checklist validated with the Delphi technique and a written self-assessment questionnaire. Results: Seventeen students (57\%) accepted to participate, corresponding to $14.3 \%$ of the cohort. Tracheal intubation was achieved by 64\%, with 60 seconds as the mean time of apnea. Less than half of the students checked the instruments, performed pre-oxygenation or evaluated the airway. Eighty-seven percent of the students said that the current curriculum offers them minimal competencies for airway management in a real clinical situation and all prefer simulated scenarios for the acquisition of clinical skills. Conclusions: The methodology currently used to teach tracheal intubation is not assuring the acquisition of the competencies to the students in this curriculum stage. More effective teaching methods are required, and the use of simulated scenarios can be a useful tool.

(Rev Med Chile 2011; 139: 165-170).

Key words: Education, medical, undergraduate; Intubation, endotracheal; Manikins.

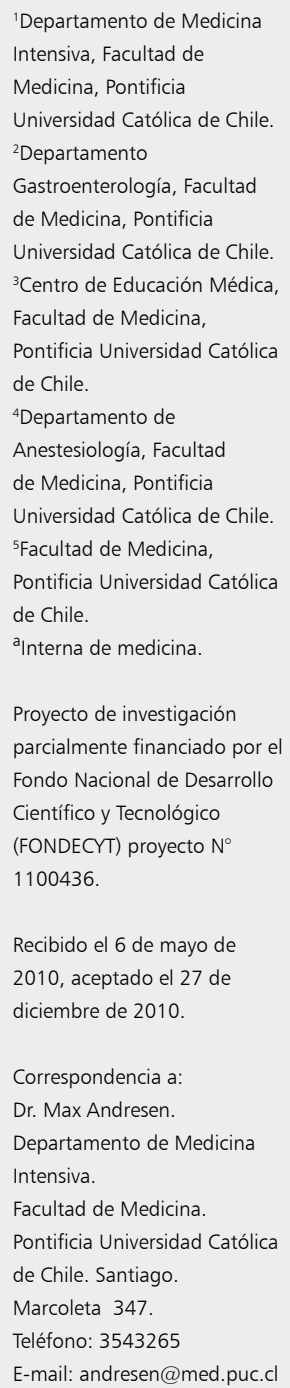<smiles>[Tl]</smiles>

as escuelas de medicina de Chile han incorporado conceptos innovadores en el campo de la educación médica, con el fin de mejorar los procesos de enseñanza y aprendizaje de sus alumnos. La Escuela de Medicina de la Pontificia Universidad Católica de Chile (EMPUC) ha realizado una reforma continua de su currículo, donde destacan la reducción del currículo obligatorio, aumento progresivo de los electivos, prácticas ambulatorias, incorporación de metodologías de enseñanza en pequeño grupo y el uso de instrumentos de evaluación que incluyen Exámenes Clínicos Estructurados (ECOE) para la evaluación de destrezas clínicas y recientemente el uso de Portafolios para evaluar reflexión en la práctica clínica y profesionalismo ${ }^{1,2}$. Sin embargo, este proceso debe considerar instancias de enseñanza y evaluación de destrezas en ambientes controlados ${ }^{3}$.

La aproximación más racional por parte de las escuelas de medicina en Chile a la adquisición de conocimientos específicos, destrezas clínicas y actitudes relacionadas con el profesionalismo, es a través de la creación de currículos basados en competencias y que, en su esencia, permiten libertad y autonomía a cada escuela de utilizar los métodos de enseñanza e instrumentos de evaluación que 
consideren pertinentes para asegurar el logro de dichas competencias terminales, ya que el Examen Médico Nacional se limita a la medición de conocimientos teóricos ${ }^{4-6}$. En los Estados Unidos de Norteamérica, se establecieron las competencias genéricas de los egresados por parte del Accreditation Council for Graduate Medical EducationACGME) ${ }^{7}$. En Chile, la Asociación de Facultades de Medicina de Chile (ASOFAMECh) identificó 93 competencias y dentro de éstas, la intubación oro-traqueal fue considerada una competencia terminal e indispensable. Su importancia se basa en la relevancia dentro de los procedimientos prácticos, así como su relación con otras competencias dependientes del manejo de urgencias, otorgamiento de consentimiento informado en procedimientos electivos y administración de fármacos en la realización de soporte vital avanzado ${ }^{8,9}$.

La enseñanza actual del manejo de la vía aérea, a lo largo de la carrera de Medicina, está basada en un modelo tutorial y suele ser más teórica que práctica, con una metodología no siempre estandarizada, que asegure la adquisición transversal de esta habilidad en todos los graduados ${ }^{10}$. A esto se suma la dificultad de la enseñanza/aprendizaje de la maniobra en situaciones de urgencia real en la práctica de los alumnos y los reparos éticos relacionados al aprendizaje tutorial durante una situación de urgencia incluyendo el riesgo vital para el paciente.

Los estudiantes de la EMPUC reciben los conocimientos relacionados con los reparos anatómicos, uso de drogas e indicaciones de reanimación cardio-pulmonar dentro de los primeros 5 años de carrera y no existen instancias de entrenamiento formal durante el internado de sexto año.

Este estudio tuvo como objetivo la evaluación formativa de las competencias relacionadas con la intubación traqueal de alumnos de sexto año de la EMPUC en un escenario simulado.

\section{Metodología}

Estudio observacional prospectivo empleando maniquí dentro de un escenario simulado de intubación traqueal. Se seleccionó en forma aleatorizada a $25,2 \%$ de la cohorte de internos de sexto año medicina, para participar en forma voluntaria en una evaluación formativa de intubación traqueal. Todos los alumnos que accedieron a participar firmaron previamente un consentimiento informado.

\section{Escenario}

Se generó un escenario en una sala equipada con los elementos necesarios para la realización de una reanimación avanzada en la UCI del Hospital Clínico de la Pontificia Universidad Católica de Chile. Se empleó un maniquí de simulación avanzado (Megacode VitalSim ${ }^{\mathrm{TM}}$, Laerdal), Mallampati grado I. El equipamiento incluyó la disponibilidad de monitoreo de electrocardiograma, monitoreo de saturación de oxígeno, equipo de aspiración, carro de paro, bandeja de intubación a la vista con: laringoscopios, tubos traqueales de distinto tamaño, jeringas, introductores maleables, ambú, fonendoscopio, material de fijación del tubo, drogas sedantes y relajantes musculares. Una auxiliar de enfermería estuvo presente para asistir a los participantes cuando le eran requeridos los materiales o drogas. El sistema se programó para mostrar desaturación de oxígeno progresiva a partir de los 60 segundos de apnea y compromiso hemodinámico a partir de los 120 segundos de apnea.

Antes de empezar se explicó a cada alumno que sería sometido a un escenario de simulación mediante el uso de un maniquí con el objetivo de evaluar las competencias requeridas en el manejo de la vía aérea e intubación traqueal electiva.

\section{Evaluación}

Un equipo de 6 expertos, compuesto por médicos intensivistas y anestesistas, confeccionó una pauta de evaluación validada mediante técnica Delf ${ }^{11}$. En la primera ronda, el panel de expertos identificó 14 aspectos de importancia en la pauta de evaluación: 1) uso de precauciones universales; 2) consentimiento informado (consulta de existencia);3) revisión del equipo e instrumental; 4) evaluación de la dificultad de la vía aérea; 5) pre-oxigenación adecuada; 6) elección de drogas y sus dosis correctas; 7) tiempo de apnea durante la intubación; 8) logro de la intubación traqueal número de intentos; 9) infla manga (cuff) del tubo traqueal; 10) evaluación subjetiva de la técnica de intubación; 11) evaluación clínica de la posición del tubo tras la intubación; 12) fijación del tubo traqueal; 13) solicita ayuda y 14) re-oxigena al paciente. Luego, mediante técnica de consenso se estableció en la segunda ronda que 10 de ellas serían considerados como aspectos de mayor im- 
portancia: $1,2,3,4,5,6,8,11$ y 12 . Sólo el punto 8 (intubación traqueal) fue considerado como crítico. La pauta utilizó una escala dicotómica (logrado-no logrado). El evento fue registrado en video para ser posteriormente evaluado mediante la pauta validada por un observador experto, enfocándose en los puntos previamente expuestos.

\section{Cuestionario de auto-evaluación}

Al finalizar el escenario simulado, los alumnos completaron un cuestionario con 4 preguntas abiertas de percepción sobre su capacitación previa en intubación traqueal y el escenario de evaluación recién realizado. Adicionalmente se creó una pauta con 5 ítems de auto-evaluación utilizando una escala Likert del 1 al 7 (1: más bajo o muy deficiente y 7: más alto o excelente) en referencia al grado de conocimientos teóricos como habilidades manuales relacionadas a la intubación traqueal, exposición a situaciones reales similares a la simulada en este trabajo en la que los participantes tuvieron posibilidad de intubar la tráquea, a la adquisición de competencias para manejar una vía aérea difícil y a liderazgo del grupo.

\section{Resultados}

Se seleccionaron en forma aleatoria a 30 alumnos que hubieran finalizado las rotaciones obligatorias correspondientes al internado de sexto año del año 2009. La muestra seleccionada corresponde a $25,2 \%$ de esta cohorte de internos (30 de 119). Diecisiete de 30 (56,7\%) alumnos invitados aceptaron participar en esta evaluación formativa, correspondiente a $14,3 \%$ de la cohorte. Todos los alumnos firmaron consentimiento informado previo al inicio de la evaluación.

\section{Evaluación}

Durante la simulación, $41 \%$ de los alumnos solicitaron el documento de consentimiento informado. El equipo e instrumental fue revisado antes de la intubación por 5,8\% de los participantes. Ninguno de ellos utilizó medidas de precaución universal. La vía aérea (dificultad de la vía aérea) fue evaluada en $23,5 \%$ de los casos. Se pre-oxigenó adecuadamente al paciente en $41 \%$ de los casos. El 29,4\% de los participantes demostraron conocimiento de los fármacos utilizados durante la intubación, incluyendo nombre, dosis y vía de administración (Figura 1).

La intubación traqueal fue lograda por $64 \%$ de los alumnos (promedio 2 intentos por operador r: 1-3). Sin embargo, $27 \%$ de ellas fueron monobronquiales. El promedio de apnea durante cada maniobra de intento de intubación fue de 60 segundos (45-146 segundos). La verificación de la posición del tubo y la fijación del tubo fue realizada en $64,7 \%$ y 52,9\% de los casos, respectivamente. Sólo 5,8\% de los participantes solicitó ayuda o respaldo de un operador más experimentado en algún momento del escenario.

\section{Cuestionario de auto-evaluación}

El $82,7 \%$ de los participantes se sintieron cómodos con el empleo del escenario simulado. Todos respondieron que preferirían la incorporación de instancias prácticas simuladas en manejo de vía aérea como estrategia para mejorar la adquisición de competencias en intubación traqueal. El 87\% de los participantes respondieron sentir

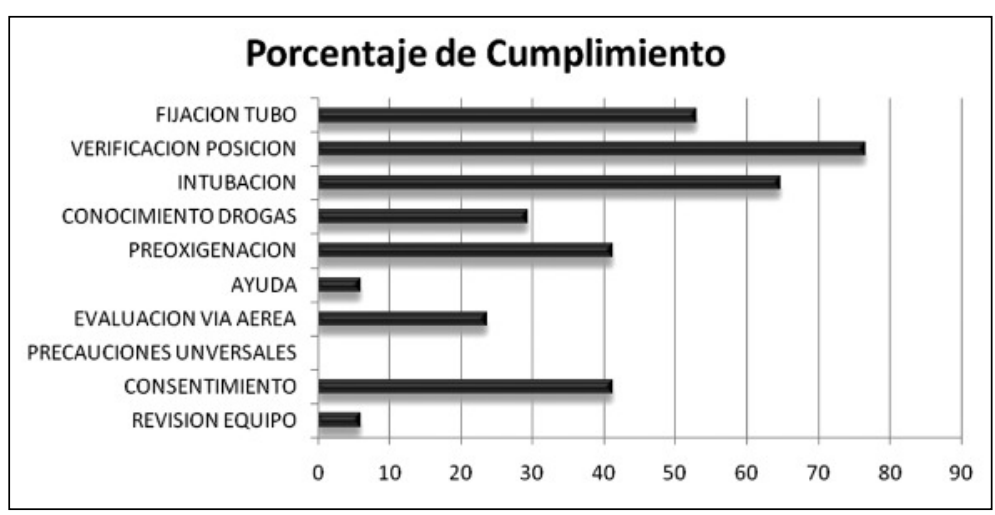

Figura 1. Porcentaje de cumplimiento de los 10 puntos de la pauta de evaluación: 1) revisión del equipo e instrumental; 2) consentimiento informado (consulta de existencia); 3) uso de precauciones universales; 4) evaluación de la dificultad de la vía aérea; 5) solicita ayuda; 6) pre-oxigenación adecuada; 7) conocimiento y elección de drogas y sus dosis correctas; 8) logro de la intubación traqueal-número de intentos; 9) verificación clínica de la posición del tubo tras la intubación, y 10) fijación del tubo traqueal. 
que su formación, con el internado de sexto año completo, no les permitía o les permitía parcialmente enfrentarse adecuadamente a intubaciones traqueales. Usando una escala Likert del 1 al 7 (1: más bajo o muy deficiente y 7 : más alto o excelente) el promedio de puntuación otorgado tanto al grado de conocimientos teóricos como habilidades manuales relacionadas a la intubación traqueal, adquiridos durante su formación, fue de 6 . La puntuación otorgada a la exposición a situaciones reales similares a la simulada en este trabajo en la que los participantes tuvieron posibilidad de intubar la tráquea ellos mismos fue de 6 . En cuanto a la adquisición de competencias para manejar una vía aérea difícil y a liderazgo del grupo la puntuación fue de 3 .

\section{Discusión}

El uso de escenarios simulados con un maniquí o con pacientes-actores entrenados, son técnicas de educación que permiten la enseñanza de ciertas habilidades en las mejores condiciones posibles. Por otro lado, ofrecen la posibilidad de evaluar, hacer seguimiento y optimizar el proceso de obtención y mantención de las competencias ${ }^{12}$. La intubación traqueal es un procedimiento frecuente, en especial en la atención pre-hospitalaria de urgencia, en procedimientos anestésicos y en UCIs. Este procedimiento comprende maniobras sencillas que se pueden adquirir en corto tiempo $y$, cuando es aplicada correctamente, cumple un objetivo importante y en ocasiones, vital ${ }^{13,14}$. El correcto manejo de la vía aérea es una competencia esencial para médicos y otros profesionales de la salud.Es por esto que se considera un objetivo terminal del currículo de las escuelas de medicina en Chile y el mundo ${ }^{8}$. Una técnica inadecuada está asociada a eventos adversos, que van desde lesión de la vía aérea hasta la muerte, en intubaciones traqueales electivas y de emergencia ${ }^{15,16}$.

En este estudio, más de 60\% de los participantes logró la intubación traqueal en un escenario de un paciente electivo. Adicionalmente, los períodos de apnea recomendados durante cada intento de intubación y el cumplimiento de varios de los pasos generales peri-intubación fueron efectuados en un porcentaje menor al deseado ${ }^{17}$. Estos hallazgos sugieren que, hasta el momento de la formación en que fueron evaluados, la metodología de enseñanza de manejo de vía aérea, no nos ha permitido asegurar la adquisición de esta competencia para todos los internos, lo que no significa que no adquieran la competencia en instancias posteriores. Esto es relevante, ya que en un currículo basado en competencias, las instancias de enseñanza y evaluación durante la carrera deben ser planificadas para monitorear su progresión a lo largo de la carrera y no sólo al momento del egreso ${ }^{18}$.

El proyecto del perfil de competencias comunes para los egresados de las escuelas de medicina de la ASOFAMECh, considera 4 niveles de competencia: 1: ninguno, 2: básico, 3: intermedio y 4: avanzado. ASOFAMECh considera para intubación oro-traqueal un nivel intermedio o 3 , que implica que deben demostrar competencia clínica sin lograr autonomía. La justificación se basa en que el aprendizaje se logrará mediante modelos, simulaciones o en un número escaso de pacientes reales. Los estudiantes no pueden desempeñar la competencia en forma autónoma debido a restricciones legales o reglamentarias, a su complejidad o por limitación de recursos o de pacientes. Se establece en el mismo documento que los conocimientos relacionados se pueden evaluar mediante pruebas escritas; la demostración clínica mediante pautas de chequeo, observación directa o ECOEs ${ }^{8}$.

Como sucede con cualquier procedimiento o destreza clínica, el aprendizaje y mantención de la habilidad para intubar la tráquea depende de la práctica continua o mediante sesiones en laboratorios de destrezas clínicas ${ }^{19,20}$. Desafortunadamente, la exposición del alumno a distintas maniobras prácticas y su aprendizaje tutorial durante su carrera en pregrado es muy variable, determinando una heterogeneidad importante en la adquisición de la competencia entre alumnos de una misma generación, a pesar de que son sometidos a las mismas rotaciones y programas durante su carrera ${ }^{20,21}$.

Actualmente, la evaluación de alumnos de medicina de último año está más orientada a certificar aspectos cognitivos (teoría), y en la demostración de destrezas clínicas relacionadas con la entrevista clínica y examen físico durante los ECOEs. Sin embargo, existe una falencia en el entrenamiento sistemático de procedimientos prácticos establecidos por ASOFAMECh ${ }^{8}$. Es necesario contar con mecanismos de enseñanza y evaluación que permitan establecer el grado de aprendizaje, logro de objetivos y sobretodo la mantención de habilidades, para poder objetivar 
que aquellas competencias clasificadas como objetivos terminales de la carrera efectivamente sean logradas durante la formación médica ${ }^{22}$.

Los alumnos de medicina debieran contar durante su etapa formativa con instancias que les permitan desarrollar sus destrezas técnicas en ambientes adecuados, controlados, bajo estricta supervisión docente y que, adicionalmente les permitan adquirir o reforzar habilidades de liderazgo y dirección del equipo de apoyo en distinta situaciones clínicas que rodean el procedimiento en cuestión, objetivo que es mejor logrado utilizando escenarios de simulación en centros de entrenamiento de destrezas clínicas habilitados para estos efectos, con espacios curriculares protegidos y posibilidad de revisar el desempeño del alumno en forma diferida ${ }^{21-23}$.

Las dependencias físicas así como los docentes involucrados en la enseñanza de destrezas clínicas deben estar alineados con los objetivos curriculares para optimizar los recursos económicos y humanos $^{23}$. El uso de instrumentos y pautas de chequeo permiten establecer el logro de objetivos curriculares integrando los conocimientos teóricos y prácticos, con competencias actitudinales como control de la situación y liderazgo del alumno ${ }^{24-26}$. Nuestros alumnos perciben un entrenamiento insuficiente en esta etapa curricular y se manifiestan a favor de la implementación de instancias formales de entrenamiento y evaluación con ambientes simulados. En el caso particular de la intubación traqueal existen instancias curriculares posteriores a la aplicación de esta evaluación que incluyen el entrenamiento simulado en quinto año, intubación electiva de pacientes reales en rotación obligatoria de cirugía (anestesia) y el uso de maniquí en sala de reanimación durante el internado obligatorio de urgencias de séptimo año cuyo impacto se podrá objetivar en el futuro. Debemos destacar la disociación entre la percepción de los estudiantes en su autoevaluación respecto a la evaluación objetiva ya que no siempre los alumnos con mayor confianza en su capacitación presentan un desempeño adecuado por lo que recomendamos el uso de pautas de autoevaluación sólo en instancias formativas. Otra limitación del estudio se relaciona con la muestra, ya que la selección de los alumnos fue realizada en forma aleatoria pero sólo 17 de los 30 seleccionados aceptaron participar, lo que impone un sesgo respecto a la motivación de los participantes.
En conclusión, encontramos de gran utilidad el empleo de escenarios simulados. En este caso en particular nos permitió evaluar la factibilidad de su implementación, la evaluación de una competencia terminal utilizando una pauta validada y la realización de un proceso de autoevaluación por parte de los alumnos. Consideramos que en este nivel curricular, los internos que demuestran competencia pueden focalizar sus esfuerzos en el logro de otras competencias esenciales. Sin embargo, aquellos que no demostraron competencia, tienen instancias curriculares durante séptimo año para adquirir las destrezas relacionadas con la intubación oro-traqueal. Este es un paso inicial en el desarrollo de herramientas que permitan evaluar las destrezas de nuestros alumnos así como de la generación de listas de chequeos apropiados para los niveles de enseñanza ${ }^{26}$. Los resultados de escenarios simulados no siempre pueden extrapolarse al comportamiento en situaciones reales pero sin duda aportan al proceso enseñanza y aprendizaje. El aprendizaje mediante la simulación suele ser bien acogido y valorado por los alumnos. En este caso, pese a tratarse de una instancia de evaluación formativa, la mayoría de los participantes expresaron su agradecimiento y le dieron un valor educativo al ejercicio.

Agradecimientos: A Dra. Marisol Sirhan (Centro de Educación Médica, Facultad de Medicina, Pontificia Universidad Católica de Chile) por sus comentarios y colaboración.

\section{Referencias}

1. Moreno R, Velasco N. [Curricular changes in the School of Medicine of the Pontificia Universidad Católica de Chile]. Rev Chil Cir 1994; 46: 333-6.

2. Rosso P, Velasco N, Moreno R. [Undergraduate curriculum reform at the Pontifical Catholic University Medical School: aims, methodology and advance status]. Rev Med Chile 1997; 125: 796-807.

3. Epstein R. Assessment in Medical Education. N Engl J Med 2007; 356: 387-96.

4. Perfil de Conocimientos Comunes Examen Único Nacional de Conocimientos en Medicina (EUNACOM). Disponible en: http://www.emn.cl/pdf/respiratoriasB. pdf. [consultado el 08 de Noviembre del 2010].

5. Enriquez O, Mena B. Habilitación profesional. Condiciones para el aseguramiento de la calidad de la educación 
médica y condiciones para la confianza recíproca. Experiencia y visión de ASOFAMECH. Rev Med Chile 2005; 133: 483-94.

6. Miller GE. The assessment of Clinical Skills/Competence/Performance. Academic Medicine 1990; 65: s63-7.

7. Accreditation Council for Graduate Medical Education. [homepage en Internet]. Chicago: ACGME;c2000-2009. Outcome Project; Disponible en: http://www.acgme.org/ acWebsite/home/home.asp. [consultado el 8 de Noviembre de 2010].

8. Proyecto MECESUP: Desarrollo y evaluación de un perfil común del egresado de las escuelas de medicina de Chile. Disponible en: https://www.ucursos.cl/ medicina/2007/0/INNCURRME/9/material_docente/ previsualizar?id_material=138916. [consultado el 08 de Noviembre del 2010].

9. Burch VC, Nash RC, Zabow T, Gibbs T, Aubin L, Jacobs B, Hift RJ A structured assessment of newly qualified medical graduates. Med Educ 2005; 39: 723-31.

10. Pepe PE, Copass MK, Joyce TH. Prehospital endotracheal intubation: rationale for training emergency medical personnel. Ann Emerg Med 1985; 14: 1085-92.

11. Hasson F, Keeney S, Mckenna H. Research guidelines for the Delphi survey technique. J Adv Nurs 2000; 32: 1008-15.

12. Cantrell MJ, Deloney LA. Integration of standardized patients into simulation. Anesthesiol Clin 2007; 25: 37783.

13. Kovacs G, Bullock G, Ackroyd-Stolarz S. A Randomized controlled Trial on the Effect of Educational Interventions in Promoting Airway Management Skill Maintenance. Annals of Emergency Medicine 2000; 36: 301-9.

14. Ti LK, Chen FG, Tan GM, Tan WT, Tan JM, Shen L, Goy RW. Experimental learning improves the learning and retention of endotracheal intubation. Med Educ 2009; 43: 654-60.

15. Kastanos N, Estopá Miró R, Marín Perez A, Xaubet Mir A, Agustí-Vidal A.Laryngotracheal injury due to endotracheal intubation: incidence, evolution, and predisposing factors. A prospective long-term study. Crit
Care Med 1983; 11:362-7.

16. Schwartz DE, Matthaway MA, CoheN NH. Death and other complications of emergency airway management in critically ill adults. A prospective investigation of 297 tracheal intubations. Anesthesiology 1995; 82: 367-76.

17. Goldmann K, Ferson DZ. Education and training in airway management. Best Pract Res Clin Anaesthesiol 2008; 19: 717-32.

18. Harden RM. Learning outcomes as a tool to assess progression. Med Teach 2007; 29: 678-82.

19. Paulus F, Binnekade JM, Middelhoek P, Vroom MB, Schultz MJ. Performance of manual hyperinflation: a skills lab study among trained intensive care units nurses. Med Sci Monit 2009; 15: CR418-22.

20. Semeraro F, Signore L, Cerchiari EL. Retention of CPR performance in anaesthetists. Resucitation 2006; 68: 101-8.

21. Weksler N, Tarnopolski A, Klein M, Schily M, Rozentsveig V, Shapira AR, Gurman GM. Insertion of the endotracheal tube, laringeal mask airway and oesophagealtracheal Combitube. A 6-month comparative prospective study of acquisition and retention skills by medical students. Eur J Anaesthesiol 2005; 22: 337-40.

22. Tiah L, Wong E, Chen MF, Sadarangani SP. Should there be a change in the teaching of airway management in the medical school curriculum?. Resucitation 2005; 64: 87-91.

23. Byme AJ, Pugsley L, Hashem MA. Review of comparative studies of clinical skills training. Med Teach 2008; 30: 764-7.

24. Bucley S, Coleman J, Davison I. The educational effects of portfolios on undergraduate student learning: A Best Evidence Medical Education (BEME) systematic review. BEME Guide No. 11. Med Teach 2009; 31: 282-98.

25. Hunt EA, Shilkofski NA, Stavroudis TA, Nelson KL. Simulation: translation to improved team performance. Anesthesiol Clin 2007; 25: 301-19.

26. Hart E, Owen H. Errors and Omissions in Anesthesia: A pilot study using a pilot's checklist. Anesth Analg 2005; 101: 246-50. 